\title{
Isolated Abducens Nerve Palsy Secondary To Varicella Zoster In A Patient With H IV
}

Ellen B. Lunenfeld M D, PGY-2 Internal M edicine; John A. Walker M D

Varicella Zoster, the recrudescence of the dormant varicella virus, occurs more often in older patients and in those who are immunocompromised. H erpes Zoster ophthalmicus ( $H Z O)$ is the second most common manifestation of the disease, second only to thoracic and lumbar zoster. ${ }^{1} \mathrm{HZO}$ is estimated to account for $10 \%$ to $25 \%$ of cases. $2,3 \mathrm{HZO}$ can lead to a number of ocular complications, including proptosis, disciform keratitis, internal ophthalmoplegia, bilateral retinal detachments, Argyll-Robertson pupil, and progressive outer retinal neuropathy (PORN).1-4

Cranial nerve palsies are a known complication of varicella zoster, most often involving the third cranial nerve, although they can include the third, fourth, fifth or sixth nerves, or any combination of these. ${ }^{4}$ Extraocular muscle paralysis, while well-documented, accounts for a small percentage of cases of ocular complications of herpes zoster ophthamicus. ${ }^{3}$ Among these, isolated sixth nerve palsies are an infrequent manifestation and have not previously been reported in a patient with HIV. We report such a case.

\section{C ase report}

A 45 year-old female with a 3-year history of untreated H IV infection was admitted to the hospital after she noted a painful vesicular rash on her face, accompanied by a high fever and decreased vision. Approximately two months prior to admission, she noted a painful rash in a dermatomal distribution on left side of her abdomen, extending from mid-axillary line to her back at approximately the level of her umbilicus. She was treated with oral acyclovir with resolution of the lesions. Approximately one month prior to admission she developed a similar rash on her face. The skin lesions were bilateral, in a mandibular distribution on her left side and on a maxillary distribution on her right side. She noted associated symptoms of malaise, high fever, and decreased vision. The patient was hospital ized for management with intravenous ((IV) acyclovir. After 14 days of treatment, she was discharged with instructions to follow up at this institution's Retina- Vitreous service. There, she was diagnosed with progressive outer retinal neuropathy (PO RN ) and was begun on oral acyclovir. O ne week later, the patient's retinal disease was found to have worsened and she was admitted for more aggressive treatment.
On admission she was noted to have resolving skin lesions consistent with varicella zoster, along with bilateral involvement of her retina with progressive outer retinal neuropathy (PORN) and a right lateral rectus palsy. All other cranial nerves were intact. Her CD 4 count and viral load were 21 cells/mL and 331,486 copies/ $\mathrm{mL}$, respectively. The patient was begun on a regimen of IV foscarnet, $1920 \mathrm{mg} \mathrm{Q} 8 \mathrm{hr}$ for a total of 14 days. $0 \mathrm{n}$ the 7th day of treatment she was begun on IV ganciclovir at $5 \mathrm{mg} / \mathrm{kg}$. She was discharged on day 18 on continued ganciclovir treatment at $5 \mathrm{mg} / \mathrm{kg} / \mathrm{d}$. While her PO RN had improved somewhat during the hospital admission, her right lateral rectus paralysis persisted.

\section{Comment}

H erpes zoster ophthalmicus is a common manifestation of varicella zoster disease with a distinct set of complications. It results when the latent virus, present in the trigeminal ganglia, is reactivated. Because of the numerous delicate structures in the vicinity of the infection, many clinically significant complications can occur. ${ }^{1}$ Extraocular muscle paralysis can manifest with both primary varicella and zoster. ${ }^{2,5}$ Reports have varied with regard to prevalence of extraocular muscle paralysis among patients with $\mathrm{H} Z \mathrm{ZO}$. M arsh et al. reviewed a series of 69 patients and found an incidence of 58 cases of extraocular muscle paralysis among 146 cases of herpes zoster ophthalmicus. In this group of patients, $42 \%$ experienced CN III involvement, $44 \%$ with CN VI involvement, and $30 \%$ with CN IV involvement. I solated CN VI nerve paralysis accounted for $25 \%$ of cases. 6 Ragozzino et al. noted 6 cases of motor deficits among 55 cases of herpes zoster ophthalmicus, none of which involved the extraocular muscles. ${ }^{3} 0$ ther reports have demonstrated an $11 \%$ to $29 \%$ incidence of extraocular muscle paralysis among patients with $\mathrm{H}$ ZO .7 The order of prevalence is greatest for the third nerve and least for the trochlear nerve. ${ }^{6}$

There have been documented cases of abducens nerve paralysis in patients with herpes zoster ophthalmicus. Goldsmith documented a case of a lateral rectus muscle paralysis which resolved within 6 months. ${ }^{4} \mathrm{~N}$ emet et al documented a case of an isolated lateral rectus muscle paralysis in a case of primary varicella. ${ }^{5}$ Archambault et 
al reported six cases of herpes zoster ophthalmoplegia, two of which were cases of sixth nerve paralysis, neither one of which occurred in isolation. ${ }^{7}$ Ramsell reported a case in which the second, third fourth, fifth and sixth nerves were all involved. ${ }^{8}$ A M EDLINE review of the literature from 1966 revealed no previously reported case of HZO-related CN VI palsy associated with HIV infection.

The exact cause of $\mathrm{HZO}$-related cranial nerve palsy is unknown. There is limited documented pathology to allow definitive analysis of tissue damage. ${ }^{7}$ Perineural and perivascular inflammation occur as a consequence of herpes zoster ophthalmicus, and may account for some of the sequelae. Theories explaining possible causes of the involved neural tissue include direct damage caused by the virus, an allergic response to the viral infection, and an occlusive vasculitis triggered by the virus. ${ }^{6}$

Treatment of extraocular paralysis secondary to varicella zoster usually involves management with antivirals, such as acyclovir or famicyclovir. The natural history of the disease is slow and spontaneous resolution in the majority of patients, usually occurring between six weeks to eighteen months. ${ }^{4}$ There is no documentation to indicate whether patients with acquired immune deficiency syndrome have a longer recovery time, or if in fact their extraocular muscle paralysis is unlikely to resolve at all. C ertainly cases of herpes zoster in H IV patients have been shown to be more severe and debilitating, presumably relating to the immunologic status of the patient. ${ }^{10}$ It is unknown, therefore, whether treatment should be directed differently towards these patients. Two months after discharge, our patient reported improved vision, but her right abducens nerve paralysis still had not resolved.

\section{References}

1. Pavan-Langston D. H erpes Zoster O phthalmicus. N eurology 1995;45(12 Suppl.8):S50-1.

2. Cobo M. Reduction of the ocular complications of $\mathrm{H}$ erpes Zoster O phthalmicus by oral acyclovir. Am J M ed 1988;85(2A):90-3.

3. Ragozzino M W, M elton LJ, Kurland LT, Chu CP, Perry $\mathrm{H} O$. Population-Based Study of H erpes Zoster and Its Sequelae. Medicine 1982;61(5):310-316.

4. Goldsmith M O. H erpes Zoster O phthalmicus with sixth nerve palsy. Can J O phthalmol 1996;3:279.

5. Nemet P, Ehrlich D. Lazar M. Benign Abducens Palsy in Varicella. Am J O phthalmol 1974;78(5):859.

6. M arsch RJ, Dulley B, Kelly V. External ocular motor palsies in ophthalmic zoster: a review. $\mathrm{Br} J \mathrm{O}$ phthalmol 1977;61:677-682.

7. Archambault P, W ise JS, Rosen J, Polomeno RC, Auger N. H erpes Zoster O phthalmoplegia: Report of Six Cases. J Clin Neuro-O phthalmol 1988;8(3):185-191.

8. Ramsell TG. Complications of herpes zoster ophthalmicus. Am J O phthalmol 1967;63(6):1796-8.

9. Vafai A, Berger M. Zoster in patients infected with H IV : a review. Am J M ed Sci 2001;321(6):372-80. 